\title{
Tunable Charge-Density Wave Transport in a Current-Effect Transistor
}

\author{
N. Marković, M. A. H. Dohmen, and H. S. J. van der Zant \\ Department of Applied Physics and DIMES, Delft University of Technology, Lorentzweg 1, 2628 CJ Delft, The Netherlands
}

(Received 24 August 1999)

\begin{abstract}
The collective charge-density wave (CDW) conduction is modulated by a transverse single-particle current in a transistorlike device. Nonequilibrium conditions in this geometry lead to an exponential reduction of the depinning threshold, allowing the CDWs to slide at much lower bias fields. The results are in excellent agreement with a recently proposed dynamical model in which "wrinkles" in the CDW wave fronts are "ironed" by the transverse current. The experiment might have important implications for other driven periodic media, such as moving vortex lattices or "striped phases" in high- $T_{c}$ superconductors.
\end{abstract}

PACS numbers: 71.45.Lr, 72.15.Nj

The charge-density wave (CDW) state, characterized by a periodic modulation of the conduction electron density, is commonly observed in low-dimensional conductors [1]. It is found to be the ground state in various inorganic and organic materials with a chainlike structure, giving rise to remarkable electrical properties [2-4]. Similar chargeordered states ("striped phases") play an important role in high- $T_{c}$ superconductors [5] and two-dimensional electron gases in the quantum Hall regime [6].

A particularly interesting feature of the CDW state is its collective transport mode, very similar to superconductivity [7]: under an applied electric field, the CDWs slide along the crystal, giving rise to a strongly nonlinear conductivity. Since even a small amount of disorder pins the CDWs, sliding occurs only when the applied electric field exceeds a certain threshold field. The pinning mechanisms, the onset of collective motion, and the dynamics of a moving CDW are typical characteristics of the complex physics which describes a very general class of disordered periodic media [8-15]. These include a wide variety of periodic systems, as diverse as vortex lattices in superconductors and Josephson junction arrays [16-19], Wigner crystals [20], colloids [21], magnetic bubble arrays [22], and models of mechanical friction [23].

The focus of recent theoretical and experimental research on disordered periodic media has been their nonequilibrium dynamical properties. In particular, it has been predicted that moving elastic structures still experience "transverse pinning" when motion occurs transverse to the periodicity of the structure [12], which was supported by numerical simulations [24]. In the context of CDW, one of the issues that has been raised is the effect of a single-particle current, due to uncondensed electrons and quasiparticle excitations. In a recent theoretical work, Radzihovsky and Toner [25] discovered that a singleparticle current has the most profound effects when it flows perpendicular to the CDW sliding direction. Based on general symmetry principles, this leads to nonequilibrium CDW dynamics even if CDW itself is stationary. Here we report our study of the CDW transport in the presence of such a transverse single-particle current.
We find that the sliding CDW motion is stable against a small transverse current, but large currents have a dramatic effect: the longitudinal depinning threshold field is exponentially reduced for normal current densities which exceed some crossover value $J_{c}$. In other words, the collective longitudinal current is enhanced by the transverse single-particle current. The characteristics of this current-effect transistor are in excellent agreement with the predictions [25].

The experiments were carried out on single crystals of $\mathrm{NbSe}_{3}$. This material has a very anisotropic, chainlike structure [2]. It exhibits two CDW transitions, each involving different types of chains, at $T_{P}=145 \mathrm{~K}$ and $T_{P}=$ $59 \mathrm{~K}$. A small portion of the conduction electrons remains uncondensed, providing a metallic single-particle channel.

A single crystal of dimensions $2.7 \mathrm{~mm} \times 36 \mu \mathrm{m} \times$ $240 \mathrm{~nm}$ was glued onto a sapphire substrate. A pattern of gold contacts was then defined on top of it using electronbeam lithography. The pattern consisted of two current leads at two ends of the crystal and a row of devices, each with two transverse current leads and two voltage leads. A scheme of such a transistor device is shown in the inset of Fig. 1.

The transverse current leads were 5-100 $\mu \mathrm{m}$ wide, and overlapped the crystal by $1-5 \mu \mathrm{m}$. To ensure contact on both sides of the crystal, a $180 \mathrm{~nm}$ thick layer of gold was evaporated at angles of $45^{\circ}$ with respect to the substrate, as well as perpendicular to it. The contact resistance of the transverse leads was by 1-2 orders of magnitude larger than the resistance of the crystal in the longitudinal direction, which precludes considerable shunting of the current through the transverse leads. A dc current of up to $1 \mathrm{~mA}$ was injected through the transverse leads. The transverse leads were not electrically connected to the longitudinal circuit, except through the crystal. Since the CDWs can slide only in the longitudinal direction, the transverse current is due to single electrons.

The longitudinal current was injected at the two far ends of the crystal. The voltage leads were $180 \mathrm{~nm}$ thick, $5 \mu \mathrm{m}$ wide, and the spacing between them was 50-500 $\mu \mathrm{m}$. The longitudinal current-voltage characteristics and the 


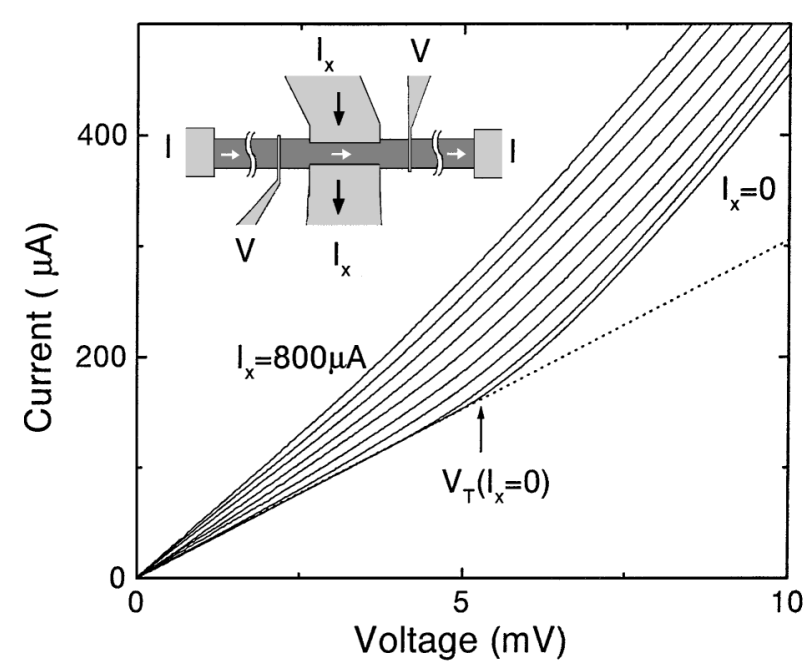

FIG. 1. Current-voltage characteristics for a $36 \mu \mathrm{m}$ wide and $0.24 \mu \mathrm{m}$ thick $\mathrm{NbSe}_{3}$ crystal at $45 \mathrm{~K}$ with the values of the transverse current of $0,100,200,300,400,500,600,700$, and $800 \mu \mathrm{A}$ (from bottom to top). The width of the transverse current leads was $100 \mu \mathrm{m}$, and the spacing between the voltage leads was $225 \mu \mathrm{m}$. The dotted line represents the Ohmic behavior of the uncondensed electrons. The data deviate from that line when the charge-density waves are depinned and start to slide, contributing additional current. The depinning threshold $V_{T}$, marked by an arrow, decreases when a transverse current $I_{x}$ is applied. Inset: The scheme of the transistorlike device, which consists of the crystal (dark shaded area) and six gold leads (light shaded areas): I for the longitudinal current, which is injected at the far ends of the crystal, $V$ for voltage measurements, and $I_{x}$ for the transverse current injection.

differential resistance were studied as a function of transverse current at different temperatures, ranging from $25-120 \mathrm{~K}$.

The current-voltage characteristics for one of the devices are shown in Fig. 1. In the absence of a transverse current, CDWs are pinned at low bias voltages. The $I-V$ is linear, as the current is due to uncondensed electrons and quasiparticles that are thermally excited above the CDW gap. When the applied voltage reaches the threshold value $V_{T}\left(I_{x}=0\right)$, marked by an arrow in Fig. 1, the CDWs are depinned and start to slide. A sharp increase in the current is observed at $V_{T}$ due to this additional conduction channel.

When a transverse current $I_{x}$ is applied, $V_{T}$ decreases and sliding starts at lower bias voltages. Thus, CDWs that were pinned for $I_{x}=0$ start sliding at lower fields when a transverse current is applied. At low bias voltages, a new linear regime appears in the $I-V$ characteristics as illustrated in Fig. 1. The resistance in this regime is lower than the single-particle resistance $R$ at $I_{x}=0$. This makes the effect easily distinguishable from heating: since most of the measurements were carried out at the temperatures at which $d R / d T>0$, heating would result in a higher single-particle resistance.

The threshold field reduction is more strikingly visible in the differential resistance measurements, shown in
Fig. 2. The differential resistance at low bias fields is mostly unaffected by the transverse current. The onset of CDW sliding, characterized by a sharp drop in differential resistance, is shifted towards zero as $I_{x}$ is increased. The same reduction of the threshold field is also observed for negative bias voltages and the plots are nearly symmetric around $V=0$. We have found no differences when changing the sign of either the longitudinal current or the transverse current.

The reduction of the sliding threshold does not occur for arbitrarily small transverse currents. The dependence of the threshold field $E_{T}$ on the transverse current density $J_{x}$ for two samples [26] is shown in Fig. 3. It is evident that $E_{T}$ remains unchanged until $J_{x}$ reaches some crossover value $J_{c}$. For $J_{x}>J_{c}, E_{T}$ decreases with increasing $J_{c}$. The transverse current density dependence of the threshold field $E_{T}$ for $J_{x}>J_{c}$ can be fit by [25]

$$
E_{T}\left(J_{x}\right)=E_{T}(0) \frac{J_{x}}{J_{c}} \exp \left(1-\frac{J_{x}}{J_{c}}\right),
$$

where $E_{T}(0)$ is the threshold field at $J_{x}=0$. Once the crossover value of the transverse current $J_{c}$ is exceeded, the depinning threshold field decreases and the CDW conduction channel is activated by much lower bias voltages.

There is a possibility that the threshold field reduction is due to current inhomogeneities around the transverse contacts. We believe that this is unlikely, for the following reasons. If no additional phase slip centers [27] are introduced between the voltage leads, then the changes in $E_{T}$ would be apparent at any value of $J_{x}$, and no $J_{c}$ would be observed. If there are additional phase slip centers, then a

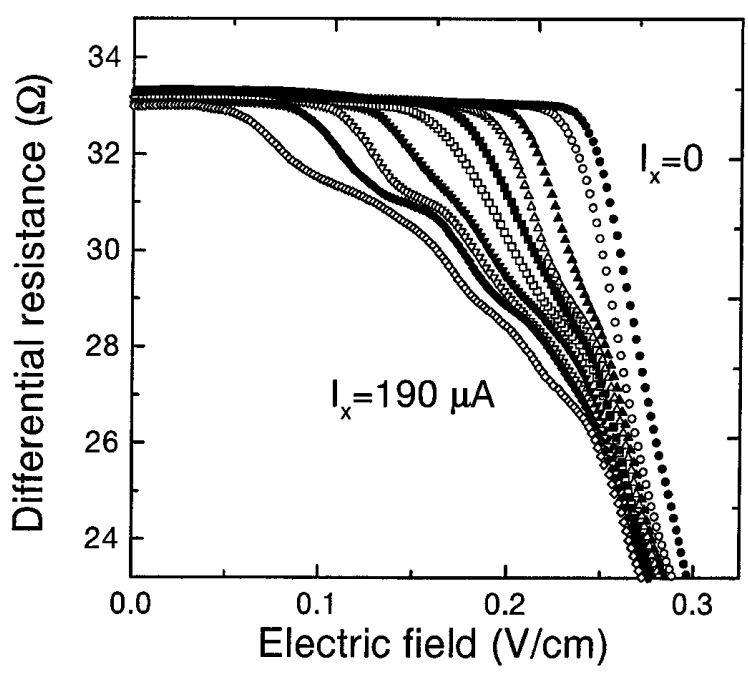

FIG. 2. Differential resistance as a function of electric field at $45 \mathrm{~K}$ of the same device as in Fig. 1. Different symbols represent different values of the transverse current $I_{x}$ : 0 (filled circles on the right), $75,100,110,120,130,150,160,170$, and $190 \mu \mathrm{A}$ (open diamonds on the left). The threshold field at which the differential resistance drops due to the depinning of the charge-density waves is shifted to lower values as the transverse current is increased. 


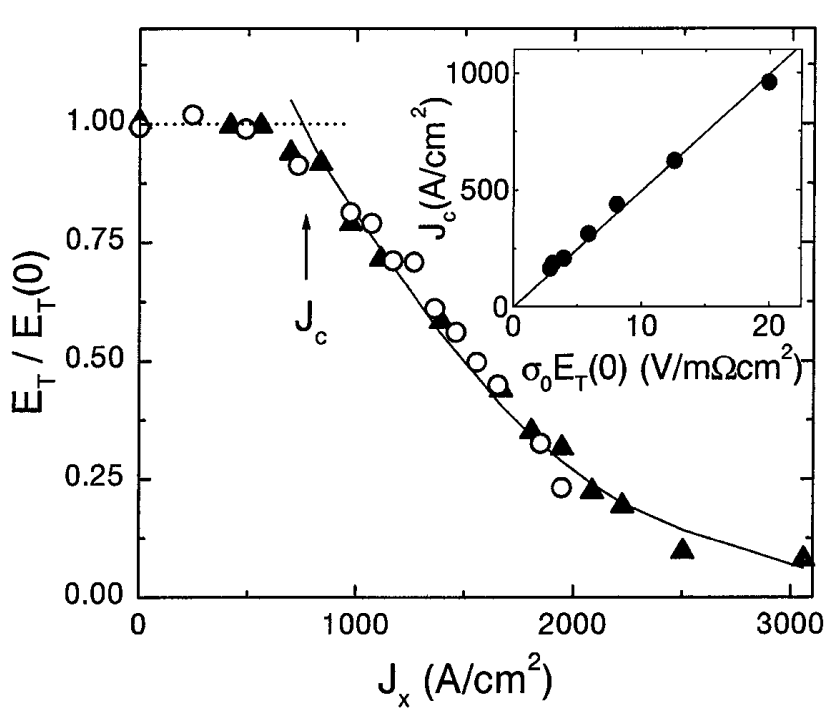

FIG. 3. Depinning threshold field $E_{T}$, scaled by its value at $J_{x}=0$, as a function of transverse current density $J_{x}$ at $55 \mathrm{~K}$ for two different devices. The widths of the transverse current leads were $100 \mu \mathrm{m}$ (circles) and $30 \mu \mathrm{m}$ (triangles). The solid line represents a fit to Eq. (1). Inset: The crossover transverse current density $J_{c}$ needed for the initial suppression of the depinning threshold $E_{T}$ increases with the value of $E_{T}$ at $J_{x}=0$. $J_{c}$ is plotted here as a function of $\sigma_{0} E_{T}(0)$ for comparison with Eq. (2), where $\sigma_{0}$ is the conductance at bias fields much larger than $E_{T}$. Each data point was determined at a different temperature: from right to left, the temperatures were $25,30,35,40$, 45,50 , and $55 \mathrm{~K}$.

nonzero $J_{c}$ may be possible as a consequence of the phase slip voltage $V_{\mathrm{ps}}$ needed to depin a small segment of the sample [28]. One would then, however, expect $J_{c}$ to be proportional to $V_{\mathrm{ps}}$, which is not the case in our experiment [29]. Furthermore, it is not clear that the current inhomogeneities would lead to the observed exponential reduction of $E_{T}$, as described by Eq. (1).

The exponential decrease of the threshold field described by Eq. (1) has recently been predicted [12,25]. In the model of Radzihovsky and Toner [25], the value of the crossover current density $J_{c}$ needed for the initial suppression of $E_{T}$ is expected to be proportional to the value of the threshold field at $J_{x}=0$, and is given by

$$
J_{c} \propto \sigma_{0} E_{T}(0)\left(\xi_{L} k_{F}\right)\left(\rho_{n} / \rho_{C D W}\right),
$$

where $\sigma_{0}$ is the conductivity at very high bias fields, $k_{F}$ the Fermi wave vector, and $\rho_{n}$ and $\rho_{\mathrm{CDW}}$ are normal and CDW electron densities, respectively. The correlation length $\xi_{L}$ $[8,30]$ is a measure for the coherence in the sample and decreases with increasing disorder.

For $T<45 \mathrm{~K}$, the temperature dependence of $E_{T}$ follows $E_{T}=E_{T}(0) e^{-T / T_{0}}$ [31], where $T$ is the temperature, and $T_{0}$ is a constant. The dependence of $J_{c}$ on $E_{T}(0)$ can therefore be studied by measuring at different temperatures. The dependence of $J_{c}$ on $\sigma_{0} E_{T}(0)$ is shown in the inset of Fig. 3: $J_{c}$ grows linearly with $\sigma_{0} E_{T}(0)$ and it extrapolates to zero for $E_{T}(0)=0$. The crossover current densities of $10^{3}-10^{4} \mathrm{~A} / \mathrm{cm}^{2}$ estimated from Eq. (2) [25] are in excellent agreement with the values measured in our experiment.

We have shown that the conduction in the CDW channel can be enhanced by a single-particle current flowing transversely to the CDW sliding direction. This surprising behavior has been observed in samples with different geometries, at different temperatures, and in both CDW regimes of $\mathrm{NbSe}_{3}$, suggesting that it is a general property of the CDW transport.

The physical origin of this effect may be that CDWs become more ordered due to momentum transfer with transversely moving normal carriers [25]. This mechanism is illustrated in Fig. 4. In the absence of defects, the charge-density wave fronts are straight and parallel to each other (the left side of the picture). The single-particle transverse current, marked by " $a$ " on Fig. 4, can flow with little or no interaction with the CDW. In the presence of defects or impurities in the crystal, the CDW deforms to lower its energy and the wave fronts are "wrinkled" (the right side of the picture). In this case, the transversely moving electrons (" $b$ ") are more likely to be deflected. The conservation of linear momentum results in a reaction force back on the CDW. This way the CDW roughness is reduced as the CDW wave fronts are straightened out or "ironed" by the transverse current. The CDW transport across the sample is therefore more coherent and less susceptible to pinning. The lower pinning strength then leads to a lower threshold field.

Since the conduction in the CDW channel can be modulated by a current in the single-particle channel, this device in principle works as a transistor, raising a question of a possible practical application. The maximum gain observed in our experiments was $\Delta I / I_{x}=0.15$. A simple estimate from our measurements suggests that the maximum gain is proportional to $\xi_{L}^{-1}$. The gain can therefore

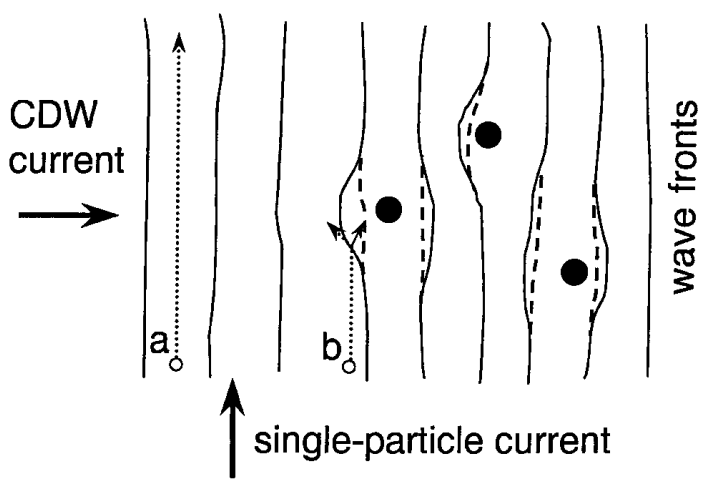

FIG. 4. Dynamical model for the threshold field reduction. The vertical lines represent the charge-density wave fronts which, if depinned, can move in the horizontal direction. The large filled circles represent impurities or other defects in the crystal. The CDW sliding direction and the transverse current direction are indicated by arrows. The small open circles (" $a$ " and " $b$ ") represent the transverse single-particle current. Deformations of the wave fronts due to momentum transfer with the transverse current are shown by the dashed lines. 
be improved by using dirtier crystals or smaller samples in which $\xi_{L}$ is limited by the sample sizes.

Apart from being intriguing in their own right as an important test of the theory, our results may provide a useful insight into related phenomena which are much more difficult to study experimentally. As mentioned above, this novel effect is relevant to a variety of other periodic systems which share the same symmetries and a similar geometry. A particularly interesting example might be the striped phases in superconducting oxides, whose role in high- $T_{c}$ superconductivity is still not resolved.

The authors are grateful to Yu. Latyshev and P. Monceau for providing the crystal, and to L. Radzihovsky, Yu. Nazarov, and S. Zaitsev-Zotov for useful discussions. This work was supported by the Netherlands Foundation for Fundamental Research on Matter (FOM). H. S. J. vdZ. was supported by the Dutch Royal Academy of Arts and Sciences (KNAW).

[1] R. E. Peierls, Ann. Phys. (Leipzig) 4, 121 (1930).

[2] For a review, see G. Grüner, Density Waves in Solids (Addison-Wesley, Reading, MA, 1994).

[3] G. Grüner, Rev. Mod. Phys. 60, 1129 (1988).

[4] R. E. Thorne, Phys. Today 49, No. 5, 42 (1996).

[5] S.A. Kivelson, E. Fradkin, and V.J. Emery, Nature (London) 393, 550 (1998); J. M. Tranquada, B. J. Sternlieb, J.D. Axe, Y. Nakamura, and S. Uchida, Nature (London) 375, 561 (1995).

[6] A. A. Koulakov, M. M. Fogler, and B. I. Shklovskii, Phys. Rev. Lett. 76, 499 (1996).

[7] H. Fröhlich, Proc. R. Soc. London A 223, 296 (1954).

[8] H. Fukuyama and P. A. Lee, Phys. Rev. B 17, 535 (1978); P. A. Lee and T. M. Rice, Phys. Rev. B 19, 3970 (1979).

[9] J. Bardeen, Phys. Rev. Lett. 42, 1498 (1979); 45, 1978 (1980).

[10] L. Sneddon, M. C. Cross, and D. S. Fisher, Phys. Rev. Lett. 49, 292 (1982).

[11] S. Scheidl and V. M. Vinokur, Phys. Rev. E 57, 2574 (1998).

[12] T. Giamarchi and P. Le Doussal, Phys. Rev. Lett. 76, 3408 (1996); P. Le Doussal and T. Giamarchi, Phys. Rev. B 57, 11356 (1998).

[13] L. Balents and M.P. A. Fisher, Phys. Rev. Lett. 75, 4270 (1995).

[14] L. Balents, M.C. Marchetti, and L. Radzihovsky, Phys. Rev. Lett. 78, 751 (1997); Phys. Rev. B 57, 7705 (1998).
[15] V. M. Vinokur and T. Nattermann, Phys. Rev. Lett. 79, 3471 (1997).

[16] G. Blatter, M. V. Feigel'man, V. B. Geshkenbein, A. I. Larkin, and V.M. Vinokur, Rev. Mod. Phys. 66, 1125 (1994).

[17] C. J. Olson, C. Reichhardt, and F. Nori, Phys. Rev. Lett. 81, 3757 (1998); C. Reichhardt, C. J. Olson, and F. Nori, Phys. Rev. B 58, 6534 (1998), and references therein.

[18] F. Pardo, F. de la Cruz, P. L. Gammel, E. Bucher, and D. J. Bishop, Nature (London) 396, 348 (1998).

[19] A. M. Troyanovski, J. Aarts, and P. H. Kes, Nature (London) 399, 665 (1999).

[20] M.-C. Cha and H. A. Fertig, Phys. Rev. B 50, 14368 (1994); E. Y. Andrei, G. Deville, D. C. Glattli, F. I. B. Williams, E. Paris, and B. Etienne, Phys. Rev. Lett. 60, 2765 (1988); F. I. B. Williams et al., Phys. Rev. Lett. 66, 3285 (1991).

[21] J. Chakrabarti, H.R. Krishnamurthy, A. K. Sood, and S. Sengupta, Phys. Rev. Lett. 75, 2232 (1995).

[22] J. Hu and R. M. Westervelt, Phys. Rev. B 51, 17279 (1995).

[23] O. M. Braun, A. R. Bishop, and J. Röder, Phys. Rev. Lett. 79, 3692 (1997); 82, 3097 (1999).

[24] K. Moon, R. T. Scalettar, and G. T. Zimányi, Phys. Rev. Lett. 77, 2778 (1996); V. I. Marconi and D. Domínguez, Phys. Rev. Lett. 82, 4922 (1999); A. B. Kolton, D. Domínguez, and N. Grønbech-Jensen, Phys. Rev. Lett. 83, 3061 (1999).

[25] L. Radzihovsky and J. Toner, Phys. Rev. Lett. 81, 3711 (1998).

[26] In the $x$ axis, the data of the $100 \mu \mathrm{m}$ device have been scaled by a factor of 0.7 . This factor can be understood from the anisotropy of $\mathrm{NbSe}_{3}$. Depending on temperature, the resistance perpendicular to the CDW chains (but still in plane) is a factor 10-20 higher than in the chain direction [N.P. Ong and J. W. Brill, Phys. Rev. B 18, 5265 (1978)]. This means that on both sides of the transverse leads current spreads out over a distance $\sim 4 W$, where $W$ is the crystal width. Note that in Fig. $3, J_{x}$ is calculated without correcting for the anisotropy of the current.

[27] N. P. Ong, G. Verma, and K. Maki, Phys. Rev. Lett. 52, 663 (1984).

[28] P. Monceau, M. Renard, J. Richard, and M. C. Saint-Lager, Physica (Amsterdam) 143B, 64 (1986).

[29] In the temperature regime of $45-55 \mathrm{~K}$, both $E_{T}$ and $J_{c}$ are almost temperature independent (see inset of Fig. 3), while $V_{\mathrm{ps}}$ changes by a factor of 4 [see, for example, M.P. Maher, T. L. Adelman, D. A. DiCarlo, J. P. McCarten, and R. E. Thorne, Phys. Rev. B 52, 13850 (1995)].

[30] A. Larkin, Sov. Phys. JETP 31, 784 (1970).

[31] J. McCarten, D. A. DiCarlo, M. P. Maher, T. L. Adelman, and R. E. Thorne, Phys. Rev. B 46, 4456 (1992). 